\title{
Preface to the Special Issue: High-Entropy Alloys
}

\author{
Yiping $\mathrm{Lu}^{1}$
}

Published online: 8 July 2020

(c) The Chinese Society for Metals (CSM) and Springer-Verlag GmbH Germany, part of Springer Nature 2020

High-entropy alloys (HEAs), novel alloys, were proposed by the Prof. Jien-Wei Yeh, in 2004. The introduction of HEAs design concept breaks the shackles and opens up a new era in the field of alloys design. More and more research reports show that HEAs have many special physical, chemical and mechanical properties Now, HEAs have become the most popular research direction in the field of metal alloys. Almost all the research institutions and universities around the world have carried out research on HEAs. HEAs have been listed by the relevant departments of the United States, the European Union and China as the key development direction in the next period. In addition to the high cost of alloying elements, the HEAs surpass the traditional alloys in mechanical properties, corrosion resistance, oxidation resistance, etc. HEAs are expected to replace all conventional alloys, regardless of element cost.

With more than 10 years of development, more and more secrets of HEAs are being revealed, and a large number of theoretical models have been established, including the composition design, solidification mechanism, deformation mechanism and corrosion resistance mechanism of HEAs. More and more work shows the uniqueness of HEAs. For example, the elements are solute and solvent atoms, and the dislocation slip is very different from that of traditional alloys. These new developments have enriched the knowledge in the field of metal alloys and challenged the existing basic theories of traditional materials science.
Chinese researchers are the first to carry out researches on HEAs. At present, Chinese researchers and American scholars are in a leading position in most of aspects of HEAs. In the research direction of HEAs, we have taken the lead, and it is one of the few important research directions in China that are in the leading position in the world. In this special issue, the microstructures, processing and properties in HEAs will be reported. Thereinto, the review on hexagonal HEAs was first published by the distinguished scholar of HEAs field, i.e., Prof. Yong Zhang from the "University of Science and Technology Beijing". Among these papers, some invited articles authored by Li et al., Pan et al., Wang et al., Zhang et al. and Cao et al. carefully address the design, microstructure, processing, mechanical, magnetic, and corrosion properties of HEAs. The subjects of the papers cover the recent major focuses of HEAs.

We sincerely thank all the authors for their noteworthy contributions and all the reviewers for their critical and constructive assessment. We also appreciate the significant assistance from the editors and the publishing team of Acta Metallurgica Sinica (English Letters) in organizing and publishing this special issue. We believe that this special issue is important and timely. The publication of this special issue would certainly generate following studies on this topic and, more importantly, benefit the alloy development of more important multi-component metallic materials.

Guest Editor Prof. Yiping Lu.

Yiping Lu

luyiping@dlut.edu.cn

1 School of Materials Science and Engineering, Dalian

University of Technology, Dalian 116024, China 


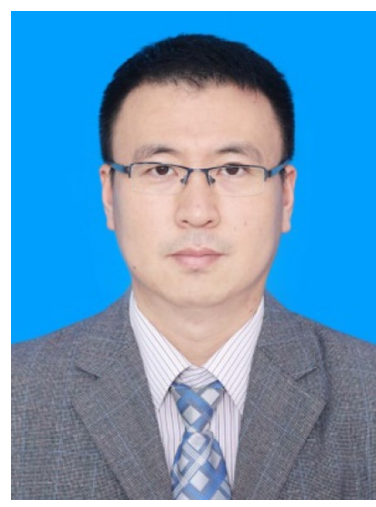

Dr. Yiping Lu is a Professor of Materials Science and Engineering at the Dalian University of Technology (DUT), China. Prof. Lu was selected as the Young and Middle-aged Innovation Leaders of the "Ministry of Science and Technology of China", the "Outstanding Youth Fund of the National Natural Science Foundation of China", the Top Youth Talents of Liaoning Province, and the Outstanding Young Scientific and Technological Talents of Dalian City. He currently serves as a committee member of several academic organization, including "Executive Director of the Youth Working Committee of Chinese Materials Research Society", "Member/ Deputy Secretary General of Solidification Science and Technology Branch of Chinese Materials Research Society", "Member of Space
Materials Science and Technology Branch of Chinese Materials Research Society", the editor of "Acta Metallurgica Sinica (English Letters)", etc. His research mainly focuses on composition design, smelting, solidified structure control, microstructural analysis and performance test of high-entropy alloys (HEAs). Dr. Lu is one of the earliest scholars to study HEAs and is also an internationally recognized leader in the fields of eutectic HEAs (EHEAs). He proposed the design idea of EHEAs and opened up a new subclass of HEAs. Dr. Lu has published $\sim 70$ peer-reviewed journal papers on HEAs with a total of over 2600 citations based on the "Google Scholar". He has written the highly cited papers, including "A promising new class of high-temperature alloys: eutectic high-entropy alloys. Scientific Reports 4 (2014) 6200"; and "Directly cast bulk eutectic and near-eutectic high-entropy alloys with balanced strength and ductility in a wide temperature range. Acta Materialia 124 (2017) 143-150”. Both articles have attracted considerable attention as illustrated by their 415 and 236 citations, respectively, searched from the "Google Scholar". These two papers are currently the highest cited paper in this EHEAs area. Dr. Lu will make further efforts to promote the development of HEAs and EHEAs. 